\title{
Arts and Science of Digital Mental Health Support
}

\author{
Marzia Hoque Tania \\ Medical Technology Research Center \\ FSE and FHEMS \\ Anglia Ruskin University \\ Chelmsford, United Kingdom \\ marzia.hoque-tania1@anglia.ac.uk
}

\author{
Ilya Andreev \\ School of Engineering and the Built \\ Environment \\ Anglia Ruskin University \\ Chelmsford, United Kingdom \\ ilya.andreev@student.anglia.ac.uk
}

\author{
Punnarumol Temdee \\ School of Information Technology \\ Mae Fah Luang University \\ Chiang Rai, Thailand \\ Punnarumol@mfu.ac.th
}

\begin{abstract}
Globally, over 600 million people are affected by mental health-related issues, according to the recent statistics. Similar to the global scenario, these issues are the single source of disease burden in the UK as well. On the bright side, the boom in the mobile application or 'app' market and the paradigm shift in the global disease burden have promoted a growing interest for mental health-related apps. This paper critically explores the mobile application for mental health support to understand what apps are available in the market and their merits and capabilities to meet the need of users' demand. This paper also provides a recommendation for future directions.
\end{abstract}

Keywords—mobile app; mental health; behavioral tracking; digital support; captology

\section{INTRODUCTION}

An average mobile phone user has $>80$ apps installed on their phone; younger demographics are more willing to pay for such apps. Therefore, not surprisingly, mobile health has become relevant from a legal and public policy point of view since 2011 [1], [2], maximizing the increasing popularity of health apps. Recently, there has been also a growing interest in the development of mental health-related apps that have paralleled the growth in smart personal devices and digital technologies. Such technologies are timely demand as there are over 600 million people affected by mental health-related issues [3]. This paper aims to critically explore the mobile apps available on the Android and iOS platform, their functionalities in behavioral tracking and mental health support, and users' reflection on these apps.

Using the key words 'mental health', 48 apps were found on the Google Play on $4^{\text {th }}$ June 2019 , which are in English. Repeating the same task on 11th June for iOS apps, resulted in 52 apps. Each platform has merits and challenges. More popular apps were found on both platforms. Under the assumption that there is no significant difference between the functionalities of the same app on both platforms, crossplatform apps were not studied twice.

\section{REACH AND TRAFFIC}

Most of the apps that meet the criteria were originated in the USA $(31 \%)$, whereas $17 \%$ of them were in the UK. However, the country of origin was not specified for more than one-fourth of these apps $(27 \%)$. The rest of the country of origin were Australia, Canada, Germany, India, Russia and
Turkey. These apps often provide a platform for online community forum to spread the support system among a wider audience [4]-[15]. In this way, the app can increase mental health awareness, and peer and expert support.

In terms of the number of downloads, Table 1 shows the most installed app to be [16], till the search date. The initial price of these apps is nil. Apart from [17], the content rating of these apps is PEGI 3, i.e. suitable for all age groups.

\section{TABLE I. APPS WITH High InSTALLATION, REVIEWS AND RATINGS}

\begin{tabular}{|l|l|c|c|c|}
\hline \multicolumn{1}{|c|}{ Name } & Install $^{\mathrm{a}}$ & Review & Rating & Ref. \\
\hline $\begin{array}{l}\text { Headspace: Meditation } \\
\text { \& Sleep }\end{array}$ & $10 \mathrm{M}+$ & 101,402 & 4.5 & {$[16]$} \\
\hline $\begin{array}{l}7 \text { Cups - Anxiety and } \\
\text { Stress Chat }\end{array}$ & $1 \mathrm{M}+$ & 15,534 & 4.2 & {$[17]$} \\
\hline $\begin{array}{l}\text { Pacifica - Stress \& } \\
\text { Anxiety }\end{array}$ & $1 \mathrm{M}+$ & 11,917 & 4.4 & {$[8]$} \\
\hline $\begin{array}{l}\text { Ada - Your Health } \\
\text { Guide }\end{array}$ & $1 \mathrm{M}+$ & 165,647 & 4.7 & {$[18]$} \\
\hline $\begin{array}{l}\text { Youper - Emotional } \\
\text { Health }\end{array}$ & $0.5 \mathrm{M}+$ & 35,433 & 4.9 & {$[11]$} \\
\hline $\begin{array}{l}\text { Moodpath - } \\
\text { Depression \& Anxiety } \\
\text { Test }\end{array}$ & $0.5 \mathrm{M}+$ & 11,711 & 4.6 & {$[19]$} \\
\hline $\begin{array}{l}\text { Self-help Anxiety } \\
\text { Management }\end{array}$ & $0.5 \mathrm{M}+$ & 42,911 & 4 & {$[20]$} \\
\hline $\begin{array}{l}\text { Wysa: stress, sleep \& } \\
\text { mindfulness therapy } \\
\text { chatbot }\end{array}$ & $0.5 \mathrm{M}+$ & 18,218 & 4.5 & {$[7]$} \\
\hline $\begin{array}{l}\text { RR Eating Disorder } \\
\text { Management }\end{array}$ & $0.1 \mathrm{M}+$ & 5,782 & 4.7 & {$[21]$} \\
\hline $\begin{array}{l}\text { Woebot: your self-care } \\
\text { expert in CBT \& } \\
\text { mindfulness }\end{array}$ & $0.1 \mathrm{M}+$ & 3,651 & 4.6 & {$[10]$} \\
\hline
\end{tabular}

The size of the app has an impact on what kind of personal device is required, hence it often unintentionally dictates the demography of the app users. The smaller sized apps include [22]-[30], ranging from 1.2 to $5.5 \mathrm{MB}$, whereas the larger apps found to be [31]-[35], all of which were more than $100 \mathrm{MB}$. We acknowledge that the memory occupancy is associated with the functionalities as well, for example, an Artificial Intelligence (AI)-enabled complex app can require larger memory space.

More than $50 \%$ of these apps provide in-app purchase facility that can be as low as less than half a pound [36] or as high as $£ 300.0$ [11]. Some of the other apps that provide more 
features via in-app purchase, costing $>£ 50.0$ are [8], [19], [23], [37], [38].

\section{FUnCTIONALITIES AND DIGITAL SUPPORT}

The available apps are often merely an informative app $(56.25 \%)$, where such information is provided in various formats such as self-help guides by the Northumberland, Tyne and Wear NHS Foundation Trust [39], where information is dissimilated in audio, video and PDF format; latest research evidence to inform the scientific community [40]; inspirational quotes by [41]. Another way utilized to inform the users was in the form of questions and the user would be able to click onto one of these, which would redirect them to a new page that contained relevant information [4], [24], [37].

The majority of the apps also allow the users to maintain a journal (47.92\%) such as [4], [9], [23] as part of Cognitive Behavioral Therapy (CBT). The diary writing platform intended to help the user throughout the period of introspection. Such apps could be a huge resource for text mining to further support the users utilizing natural language processing to automate the detection and tracking of users' emotional journey. On the other hand, instead of expecting the users to type a block of text to record the emotional journey, apps such as [42] allow the user to select a single word that best describe their current mental state from a given category and/or provide scoring from a scale. Categories are often provided with a visual scoring using emoji [43].

Currently available apps allow a wide range of behavioral tracking. There are several apps to support people to tackle their eating disorder by food tracking [13], [21]. Calorie intake, diet composition and patterns of food consumption have a bidirectional relation with emotions. An unhealthy attitude towards food can be linked to deeper psychological issues [44]. Prevalence of caffeine and alcohol addiction promoted the app market to track such consumptions as well [8]. However, the advanced works done in the literature within this domain are yet to find their way to market [45], [46].

The increasing trend of sleeping problems over the past few decades is reflected in the app market [47]. Developers and business entities have taken several attempts to help people in better management of sleep-related problems by sleep tracking or proving a relaxation based intervention [7], [16]. Few attempts have been initiated to conduct clinical trials for such issues (e.g. insomnia) [48].

Advancement in the technological world to understand natural languages has prompted intelligent 'chatbot' that is being well utilized by the app companies [7], [10]. Awardwinning app [10], backed up with extensive research at Stanford University [49] has drawn global attention. These chatbots are already capable enough to categorize the text accumulated via an AI-enabled chatbot according to mood [10], [11] and support users in managing stress and better coping with anxiety [7]. The sense of being heard without any human judgment is one of the reasons for the popularity of these apps. However, it is human nature to long for attention by other humans. Therefore, alternatives are available providing chat functions with peers instead of bots [17], [50]. Chat functions are also available to enable conversations with a professional [50]. Apps also provides information to emotionally vulnerable population on how to seek professional help who need face-to-face intervention and therapy [5], [8], [13], [17], [20]-[22], [24], [43].

One fourth of the selected apps are comprised of mental health assessment tools for various purposes [5], [10], [11], [18], [19], [21], [22], [28], [29], [51]-[55]. For example, [28] conducts mental health assessment in order to create a network of support with family, friends and professional help in case of an emergency, whereas self-help apps by [19], [20] aim to empower users through a series of questionnaires.

Digital support is also available to facilitate a wide range of interventions. Breathing exercises are one of the most ancient methods to improve health issues. The guidance provided by NHS also contains calming breathing techniques in mitigating stress, anxiety and panic [56]. Therefore, it is not a surprise that more than one third of the apps come with breathing exercises such as [4], [11], [15], [20], [22], [24], [27], [29], [43], [55].

The interface of apps such as [20] contains a circle in the center of the screen contracting and expanding symbolizing the user's breathing pattern. The user is required to control their breathing to match the circle. The program starts off with a faster pace and gradually slows down. Others also have a timer countdown above the circle. The process can be paused and started by tapping the screen [24]. Some apps also have an audio voice over to guide the user through the exercise without having them to look at the screen [24], [27], [29].

Audio guided meditation is supported by one of the available apps such as [16], [37] to improve user's mental wellbeing via relaxation audio, calming instructions, etc. The audio guided meditations are particularly more useful to the users who want to perform these activities while commuting or being engaged with other day-to-day activities. Calming audio exercises are used as a tool to help users in reducing anxiety [20], managing stress, depression as part of a CBT program [19]. CBT based apps also allow the user to set goals or daily challenges [9], [37], [43]. [37] supports the users to overcome their negative thoughts with the help of positive psychology, mindfulness and CBT. For example, users are encouraged to perform a simple exercise such as having a positive though for any negative one. [43] tries to encourage users to accomplish important life goals and be on track.

Users can be engaged with the app via gaming activities as part of a coping mechanism, introspection, positive psychology etc. [4], [5], [12], [15], [16], [21], [27], [30], [35], [37], [43]. The apps use trivia, selection of feel-good words, role-playing, gamification tools for various lengths of time, depending on the task and requirement of the intervention. These innovative approaches to improve mental health harness the advancement in the gaming world onto the serious gaming platform.

The above functionalities are not always embedded as buttons, rather prompted by a series of actions. Apps aim to reduce emotional episodes such as stress with prompted activities such as mindfulness, deep breathing or reading various coping cards [4], [6], [11], [15], [20], [22], [24], [27], [29], [43], [55], [57]-[59]. Such actions can be enacted by a user's interaction with the app stating the current problem. A 
number of apps [4], [37] provide activities for the user to complete in order to take the mind off the stressor. Periodical prompts are also available that act as a reminder to the user to perform an action to maintain a balanced mental health [9], [10], [13], [16], [20], [24], [27], [30], [60]-[64]. However, such prompts can be turned off by users.

The currently available apps are capable to generate automatic reports [8], [13], [15], [19], [21], [22], [37], [42], [51], [55], empowering the users to be fully aware of their mental health condition, which can be shared with their conventional therapists as well.

\section{ASSESSMENT OF THE DigITAL SUPPORT}

Considering information cues provided by the app stores to users, this study is in agreement with [65], where positive relation was found for both numbers of reviews and app ratings, up to a certain extent, with the number of installations. It can be seen from Table I that the apps with hundreds of thousands of reviews also have over a million installations. However, it should be noted that the most-reviewed app in Table I is not exclusive to mental health-related issues only.

Table I also shows that the relationship between the number of app reviews or the rating is not linear. A further investigation including all apps that fit the inclusion criteria clearly indicated that despite the massive number of downloads, there are inconsistencies and lack of continuation. The high number of app downloads per day does not show how many of the users are still active. Depending on whether the app was intended for one-off use such as one-time mental health assessment, fix intervention program, or continuous support, the information cues (Table I, [65]) could be misleading. Reflecting on the app rating and comments section, it is unclear that how many users have favored the app or claimed beneficiation. Examination of these apps also found some ambiguity in how and why users are deciding about their views on these apps. [37] claimed $86 \%$ of their consistent app users to report feeling better about their lives in 2 months. Although an independent evaluation of such a claim is necessary, more quantifiable metrics such as [37] would benefit the users to make an informed decision based on statistics.

A critical exploration of 25 behavioral health apps, [66] revealed the need for standardization of the quality of such apps and a more effective metric to inform the users. [66] urged for better guidance for the apps that not only include evidence but also integrate disclosed consumer choices.

Not all the apps explored under this study were categorized as a 'Medical' app by the app store. Some of these apps also fall under the category of 'Health \& Fitness' and 'Lifestyle'. Therefore, technically, the makers of these apps may subside medical device compliance. Despite the attempts taken to develop a more effective measure to evaluate the mobile health apps, there is no existing universally accepted systematic framework for the users to make an informed decision about these apps [67]-[69]. More useful framework may result in as an outcome of the soon to be in action, Medical Devices Regulation (MDR 2017/745).
The reported articles already demanded more observational study, validation of effectiveness, clinical trial and more empirical evidence [66], [70]. Our paper also finds that there is a clear gap to understand and link the need and user adoption to adequately evaluate the digital mental health support on the mobile platform.

\section{DISCUSSION}

The intended users of the mental health app are already a vulnerable group who feels a need for support. Therefore, the manufacturers of these apps should not take inconsistency and lack of information lightly. If an app is developed under a project, which is going to be discontinued after a certain period or has been discontinued, the users should be clearly warned about this information.

The manufacturers and developers need a better understanding of the need of these vulnerable groups from the perspective of the end-users as well as the experts. These understandings are not limited to the mental health condition itself, but also how people who need mental health support use these apps to gain a benefit, how and why they drop-out, what it signifies in terms of their well-being. The app producers should not generalize the lack of known harm to a single condition as an entirely null effect, because 'no known adverse effect for a single condition' may not be applicable or validated for digital behavior.

Similar to conventional learning styles, the learning pattern via virtual media also varies from person to person. The benefit of the digital tool is the means to quantify as well as the power to adjust for the variation. More insights on virtual learning style for any given situation is yet to be discovered. Person-specific mental health condition adds another dimension to the variability in learning styles. 'Personalization' using AI is the current buzz word in the mental health app market. Another observation of this paper is regarding the degree of personalization. A framework to assess the mental health apps should also comprise metrics to evaluate the functionalities such as personalization- how personalized the app is, relevance to the mental health condition, the impact of this personalization on the targetcondition and general well-being of the user.

Future researchers, developers, and people who need support for their mental health may benefit from a comprehensive mental health app assessment tool that is capable to quantify all the relevant dimensions as discussed in the paper.

\section{REFERENCES}

[1] W. H. Organization, "mHealth: new horizons for health through mobile technologies.," mHealth new horizons Heal. through Mob. Technol., 2011.

[2] N. Bol, N. Helberger, and J. C. M. Weert, "Differences in mobile health app use: A source of new digital inequalities?," Inf. Soc., vol. 34, no. 3, pp. 183-193, May 2018.

[3] World Health Organization, "Mental Health," WHO, 2019. [Online]. Available: https://www.who.int/mental_health/en/. [Accessed: 12Dec-2019]. 

Play, 2019.

[5] MoodTools, "MoodTools - Depression Aid.” Google Play, 2019

[6] Companion Approach, "Stress \& Anxiety Companion." Google Play, 2019.

[7] Touchkin, "Wysa: stress, sleep \& mindfulness therapy chatbot." Google Play, 2019.

[8] Sanvello Health Inc., "Sanvello - Stress \& Anxiety Help.” Google Play, 2019.

[9] Quirky Apps, "Quirk CBT (Cognitive Behavioral Therapy)." Google Play, 2019.

[10] Woebot Labs, "Woebot: your self-care expert in CBT \& mindfulness." Google Play; Apple, 2019.

[11] I. Youper, "Youper - Emotional Health." Google Play; Apple, 2019.

[12] My Possible Self Ltd, "My Possible Self: The Anxiety \& Mental Health App." Google Play, 2019.

[13] Recovery Warriors, "Rise Up: Eating Disorder Help." Google Play, 2015.

[14] Expert Self Care Ltd, "Student Health App." Google Play, 2019.

[15] Thrive Therapeutic Software Ltd, "Thrive: Mental Wellbeing." Google Play, 2019.

[16] M. and S. \& F. Headspace for Meditation, "Headspace: Meditation \& Sleep." Google Play, 2019

[17] 7 Cups of Tea, "7 Cups: Anxiety \& Stress Chat." Google Play, 2019

[18] Ada Health, “Ada - your health companion.” Google Play; Apple, 2019.

[19] MindDoc Health, "Moodpath - Depression \& Anxiety Test." Google Play, 2019.

[20] University of the West of England, "Self-help Anxiety

Management.” Google Play, 2017.

[21] Recovery Record, "RR Eating Disorder Management." Google Play.

[22] SilverCloud Health, "SilverCloud." Google Play; Apple, 2019.

[23] MoodTools, "CBT Thought Diary." Google Play, 2019.

[24] MoodTools, "PanicShield - Panic Attack Aid." Google Play, 2018.

[25] happtic Pty. Ltd., "Thought Diary Pro on the App Store." Apple, 2014.

[26] SSSFT NHS, "Veterans Mental Health." Apple, 2015.

[27] stem4, "Calm Harm - manages self harm." Google Play, 2019.

[28] MiDS d.o.o., "iHELP Family." Apple, 2015.

[29] MoodTools, "FearTools - Anxiety Aid." Google Play, 2019.

[30] L. SuperBetter, "SuperBetter." Google Play, 2019.

[31] B. W. Ltd, "Apart of Me." App, 2019.

[32] SSSFT NHS, “Joining Forces." Apple, 2015.

[33] inward inc, "Mindfulness Daily." Apple, 2019

[34] TalkLife Limited, "TalkLife: Depression \& Anxiety.” Apple, 2019.

[35] Remente, "Remente - Self Improvement." Apple, 2015.

[36] Lewis \& Palmer Ltd., "Clementine." Apple, 2018

[37] I. Happify, "Happify." Google Play, 2019.

[38] Mindset Health Pty Ltd, "Mindset: Hypnotherapy \& Sleep." Apple, 2019

[39] NTW NHS Foundation Trust, "Mersey Care Self Help." Google Play, 2018.

[40] The British Psychological Society, "Research Digest." Google Play, 2018

[41] Squarevibe Inc., "Anxiety No More.” Apple, 2015.

[42] eMoods, "eMoods Bipolar Mood Tracker." Google Play, 2019.

[43] Anxiety Canada Association, "MindShift CBT - Anxiety Canada." Google Play, 2019.

[44] S. J. Spencer, A. Korosi, S. Layé, B. Shukitt-Hale, and R. M. Barrientos, "Food for thought: how nutrition impacts cognition and emotion," npj Sci. Food, vol. 1, no. 1, Dec. 2017.

[45] S. Hassanpour, N. Tomita, T. DeLise, B. Crosier, and L. A. Marsch, "Identifying substance use risk based on deep neural networks and Instagram social media data," Neuropsychopharmacology, vol. 44, no. 3, pp. 487-494, Feb. 2019

[46] S. Yang, P. Zhou, K. Duan, M. S. Hossain, and M. F. Alhamid, "emHealth: Towards Emotion Health Through Depression Prediction and Intelligent Health Recommender System," Mob. Networks Appl., vol. 23, no. 2, pp. 216-226, Apr. 2018.

[47] S. Bhaskar, D. Hemavathy, and S. Prasad, "Prevalence of chronic insomnia in adult patients and its correlation with medical comorbidities," J. Fam. Med. Prim. Care, vol. 5, no. 4, p. 780 2016.

[48] Clinical Trials, "Validation of the Dayzz Digital Sleep Training App in Insomnia and Sleep Apnea," U.S. National Library of Medicine, 2019. [Online]. Available:

https://clinicaltrials.gov/ct2/show/NCT03955120. [Accessed: 17 Dec-2019]

[49] K. K. Fitzpatrick, A. Darcy, and M. Vierhile, "Delivering Cognitive Behavior Therapy to Young Adults With Symptoms of Depression and Anxiety Using a Fully Automated Conversational Agent (Woebot): A Randomized Controlled Trial.," JMIR Ment. Heal., vol. 4, no. 2, p. e19, Jun. 2017.

[50] INVOLTA INC, "PSY - mental health help. Support groups." Google Play, 2019.

[51] I. Mind Diagnostics, "Mental Health Tests." Google Play, 2019.

[52] Mark Grant, "Anxiety Release based on EMDR.” Google Play, 2019.

[53] C. P. A. R. Inc., "Bust PTSD." Google Play, 2019

[54] Carlos Gonzalez, "MHCT." Apple, 2019.

[55] US Department of Veterans Affairs (VA), "PTSD Coach." Google Play, 2019.

[56] N. Health A to Z, "Breathing exercise for stress," NHS, 2018. [Online]. Available: https://www.nhs.uk/conditions/stress-anxietydepression/ways-relieve-stress/. [Accessed: 17-Dec-2019].

[57] Grassroots Suicide Prevention, "Stay Alive." Google Play, 2019.

[58] Blue Step Solutions, "WellMind." Google Play, 2017.

[59] Dr Amanda Commons Treloar, "MyPsyDiary Pro: Track \& Improve Mental Health." Google Play, 2016.

[60] meemo-tec OG, "UP! - Depression, Bipolar \& Borderline Management." Google Play, 2019.

[61] Online Therapy $\mid$ Chat with a Therapist, "Therapy - Professional Mental Health Sessions.” Google Play, 2019.

[62] ArtSmart Software, "PsychApp - Depression, Anxiety, Panic Attack." Google Play, 2016.

[63] 365 Positivity, "Mental Health Recovery Guide." Google Play, 2015.

[64] UC San Francisco, "UCSF PRIME." Google Play, 2018.

[65] H.-Y. Huang and M. Bashir, "Users' Adoption of Mental Health Apps: Examining the Impact of Information Cues.," JMIR mHealth uHealth, vol. 5, no. 6, p. e83, Jun. 2017.

[66] A. D. Carlo, R. Hosseini Ghomi, B. N. Renn, and P. A. Areán, "By the numbers: ratings and utilization of behavioral health mobile applications," npj Digit. Med., vol. 2, no. 1, Dec. 2019.

[67] M. Neary and S. M. Schueller, "State of the Field of Mental Health Apps," Cogn. Behav. Pract., vol. 25, no. 4, pp. 531-537, Nov. 2018

[68] American Psychiatric Association (APA), "App Evaluation Model." [Online]. Available:

https://www.psychiatry.org/psychiatrists/practice/mental-healthapps/app-evaluation-model. [Accessed: 26-Dec-2019].

[69] A. Baumel, K. Faber, N. Mathur, J. M. Kane, and F. Muench,

"Enlight: A comprehensive quality and therapeutic potential evaluation tool for mobile and web-based eHealth interventions," $J$. Med. Internet Res., vol. 19, no. 3, Mar. 2017.

[70] K. G. Baron, J. Duffecy, M. A. Berendsen, I. Cheung Mason, E. G. Lattie, and N. C. Manalo, "Feeling validated yet? A scoping review of the use of consumer-targeted wearable and mobile technology to measure and improve sleep," Sleep Medicine Reviews, vol. 40. W.B. Saunders Ltd, pp. 151-159, 01-Aug-2018. 